\title{
Radical Lymph Node Dissection Along the Proximal Splenic Artery During Laparoscopic Gastrectomy for Gastric Cancer Using the Left Lateral Approach
}

\author{
Shingo Kanaji, MD, PhD, Satoshi Suzuki, MD, PhD, Masashi Yamamoto, MD, PhD, Yoshiko Matsuda, MD, PhD, \\ Kimihiro Yamashita, MD, PhD, Takeru Matsuda, MD, PhD, Taro Oshikiri, MD, PhD, Tetsu Nakamura, MD, PhD, \\ Yasuo Sumi, MD, PhD, and Yoshihiro Kakeji, MD, PhD
}

Division of Gastrointestinal Surgery, Department of Surgery, Graduate School of Medicine, Kobe University, Kobe, Hyogo, Japan

\begin{abstract}
Background. Recent technical improvements allow safe laparoscopic lymph node dissection (LND) in gastric cancer. ${ }^{1,2}$ In suprapancreatic LND, careful LND around the celiac artery (CA) is essential. From a patient's right side, deep LND is performed around the right side of the CA after dissecting around the common hepatic artery (CHA). For LND around the left side of the CA on the same operative axis as the right side, we developed a new procedure for LND along the proximal splenic artery (SA), performed from the patient's left side.

Methods. After LND around the CHA and right side of the CA from the patient's right side, the surgeon then moves to the patient's left side. The anterior pancreatic fascia is cut at the middle point of the SA to discern the dorsal layer of the LN along the SA, such as the splenic vein. LND is performed by preserving the posterior pancreatic fascia around the SA in a left-to-right direction. Finally, the LNs around the left side of the CA are deeply dissected.

Results. We performed this procedure on ten patients between April 2016 and January 2017; no operative complications were reported in grade II or higher cancer patients. ${ }^{3}$ After exposing the dorsal landmark, LNs around
\end{abstract}

the proximal SA and left side of the CA were removed in all patients.

Conclusion. This procedure enables early identification of the dorsal layer and deep LND around the left side of the CA, keeping this layer. The left lateral approach is useful for radical LND along the proximal SA.

DISCLOSURE Shingo Kanaji, Satoshi Suzuki, Masashi Yamamoto, Yoshiko Matsuda, Kimihiro Yamashita, Takeru Matsuda, Taro Oshikiri, Tetsu Nakamura, Yasuo Sumi, and Yoshihiro Kakeji have no commercial associations that may create a conflict of interest in connection with any of the products mentioned in this article.

\section{REFERENCES}

1. Kanaya S, Haruta S, Kawamura Y, Yoshimura F, Inaba K, Hiramatsu $\mathrm{Y}$, et al. Video: laparoscopy distinctive technique for suprapancreatic lymph node dissection: medial approach for laparoscopic gastric cancer surgery. Surg Endosc. 2011;25:3928-3929

2. Okabe H, Obama K, Kan T, Tanaka E, Itami A, Sakai Y. Medial approach for laparoscopic total gastrectomy with splenic lymph node dissection. J Am Coll Surg. 2010;211:e1-6

3. Dindo D, Demartines N, Clavien PA. Classification of surgical complications: a new proposal with evaluation in a cohort of 6336 patients and results of a survey. Ann Surg. 2004;240:205-213
Electronic supplementary material The online version of this article (doi:10.1245/s10434-017-5877-4) contains supplementary material, which is available to authorized users.
(C) Society of Surgical Oncology 2017

First Received: 2 February 2017;

Published Online: 15 May 2017

S. Kanaji, MD, PhD

e-mail: kanashin@med.kobe-u.ac.jp 